\title{
El papel de la actividad experimental en el desarrollo de tópicos de Física Contemporánea: Estrategia de aula para la comprensión del fenómeno de la superconductividad
}

The role of experimental activity in the development of Contemporary Physics topics: Classroom strategy for understanding the phenomenon of superconductivity

\author{
Yuly Andrea Gualtero Martínez ${ }^{*} @$, Sergio J. Sciutto ${ }^{2} @$ \\ ${ }^{1}$ Colegio Bilingüe Clermont, Bogotá, Colombia. \\ ${ }^{2}$ Universidad Nacional de La Plata, CONICET, Instituto de Física La Plata, Departamento de Física, La Plata, Argentina.
}

Recibida en 08 de Julio, 2021. Revisado en 27 de Septiembre, 2021. Aceptado en 29 de Noviembre, 2021.

\begin{abstract}
Presentamos un análisis detallado de diversos aspectos del diseño, ejecución y evaluación de resultados de una estrategia de aula para la enseñanza de tópicos de Física Contemporánea a nivel medio. Esta experiencia se llevó a cabo con un grupo de estudiantes del último año de secundaria en Bogotá, Colombia, abordando el tema de la superconductividad. Utilizando el enfoque pedagógico de la Enseñanza para la Comprensión, se diseñó un plan de clases y actividades teóricas y experimentales con el objetivo de lograr la comprensión del fenómeno de la superconductividad junto con diferentes conceptos de la física clásica y contemporánea relacionados con ese fenómeno, de los avances tecnológicos actuales y las implicaciones sociales que trae el desarrollo de la ciencia en los diferentes contextos mundiales. Este plan fue puesto en práctica con resultados muy satisfactorios, que son analizados y discutidos en el presente artículo.
\end{abstract}

Palabras-clave: Superconductividad, Física contemporánea, Enseñanza para la Comprensión, Actividades Experimentales.

\begin{abstract}
We present a detailed analysis of various aspects around the design, execution and results evaluation of a classroom strategy for teaching Contemporary Physics topics at the intermediate level. This experience was carried out with a group of high school seniors in Bogotá, Colombia, addressing the issue of superconductivity. Using the pedagogical approach of Teaching for Understanding, a class plan of theoretical and experimental activities was designed with the aim of understanding the phenomenon of superconductivity together with different related concepts of classical and contemporary physics, and of current technological advances and social implications that the development of science brings in different world contexts. This plan was put into practice with very satisfactory results, which are analyzed and discussed in this article.
\end{abstract}

Keywords: Superconductivity, Contemporary Physics, Teaching for Understanding, Experimental Activities.

\section{Introducción}

La enseñanza de diferentes saberes en los niveles de educación básica y media en las instituciones educativas públicas y privadas colombianas, debe cumplir con los lineamientos curriculares establecidos por el Ministerio de Educación Nacional (MEN), el cual presenta, por áreas de conocimiento, estándares básicos de competencias que buscan que los estudiantes "no se limiten a acumular conocimientos, sino que aprendan lo que es pertinente para su vida y puedan aplicarlo para solucionar problemas nuevos en situaciones cotidianas" [1].

Particularmente, la enseñanza de la física se encuentra enmarcada en los estándares del área de ciencias naturales, desde los cuales se pretende formar a los estudiantes en habilidades como: la exploración de hechos y fenómenos, el análisis de problemas, la observación,

\footnotetext{
* Correo electrónico: andreagualtero0804@gmail.com
}

la recolección y la organización de información relevante, la utilización de diferentes métodos de análisis, la evaluación de los métodos y la socialización de los resultados. Sin embargo, al revisar dichos estándares de ciencias naturales y seleccionar aquellos que responden a la adquisición del conocimiento en el campo de la física, es posible observar que los temas que propone el MEN pertenecen a las ramas de la mecánica, termodinámica, mecánica ondulatoria y de fluidos, astronomía y electromagnetismo, vistas desde un nivel básico y elemental.

Por esta razón, en la actualidad surge la necesidad de enseñar temas de física moderna y contemporánea, los cuales han permitido entender el mundo que nos rodea: desde los procesos químicos y físicos que tienen lugar en nuestro sol, hasta los desarrollos del mundo tecnológico. En ese orden de ideas, a pesar de vivir en un mundo que funciona y que se explica con la física que se ha ido descubriendo y desarrollando desde el siglo XX, los maestros en la escuela siguen enseñando la física que 
se desarrolló hasta principios del siglo XIX, haciendo mayor énfasis en la física del siglo XVII, la cual es importante como base histórica del desarrollo científico y como base teórica para entender la física más avanzada, pero carece de conceptos que explican los fenómenos de nuestro entorno, artefactos, experimentos y desarrollos tecnológicos presentes en la cotidianidad.

A partir de lo anterior, se buscó enseñar en un colegio de Bogotá, capital de Colombia, por medio del diseño, ejecución y evaluación de una estrategia de aula, un tema de la física contemporánea, que tuvo como finalidad hacer que los estudiantes reflexionaran acerca de la importancia de entender los desarrollos científicos que se realizan en la actualidad y que tienen una relación estrecha con los avances tecnológicos que hacen que mejore la calidad de vida de las personas. Particularmente, se abordó el tema de la superconductividad con estudiantes de último año de secundaria, del Colegio Clermont, quienes en años anteriores estudiaron una amplia gama de conceptos pertenecientes a la física clásica. A continuación, se mostrarán los detalles alrededor de las acciones que se llevaron a cabo en cada una de las etapas de la investigación.

\section{Justificación}

Teniendo en cuenta que, como se mencionó en la introducción, los estándares de educación nacional para la enseñanza de la física, no incluyen el desarrollo científico que se dio a partir del siglo XX, para poder dar cuenta del impacto que la física contemporánea tiene en estudiantes de educación media, es indispensable escoger un tema que sea llamativo para los estudiantes, que no se desvincule del todo con los estándares que propone el MEN y que sea propicio para observar la relevancia de enseñar física contemporánea en un curso de secundaria. Es así como se escogió el tema de superconductividad para evaluar este impacto.

Inicialmente, surgieron dos preguntas importantes relacionadas con la pertinencia de la superconductividad para ser abordada en la implementación. La primera pregunta fue: ¿La superconductividad es un tema que corresponde a la física contemporánea? Las razones que justifican la respuesta afirmativa de esta pregunta, radica en las investigaciones que se han desarrollado desde 1911 alrededor de este fenómeno (que no se presenta espontáneamente en nuestro entorno cotidiano), los avances científicos que se han llevado a cabo de acuerdo con los descubrimientos que físicos y químicos han realizado en torno a las propiedades de estos materiales y finalmente a la necesidad de formular una nueva teoría que explique el comportamiento inusual de los superconductores de alta temperatura, ya que como lo mencionan las investigadoras del Instituto de Ciencias Aplicadas de Madrid [2, con la teoría BCS se pueden explicar los fenómenos de la superconductividad convencional a partir de las vibraciones de la red de átomos que conforman dichos materiales; pero en los superconductores no convencionales, no hay una teoría aceptada que pueda describir el fenómeno, lo cual genera que este tópico sea objeto de estudio en las investigaciones actuales.

La segunda pregunta fue: ¿Por qué se escogió el tema de la superconductividad para ser implementado en la estrategia de aula? La superconductividad tiene una ventaja por encima de otros temas de física contemporánea, que radica en la posibilidad de llevar a cabo el experimento demostrativo en el aula de clase, en lo cual cumpliría con el primer criterio que se expuso inicialmente: ser llamativo para los estudiantes. Sin embargo, resulta necesario mencionar que para poder llevar a cabo el experimento en el aula de clase se requiere contar con proveedores de materiales de laboratorio, apoyo económico por parte de la institución educativa y la preparación necesaria para la correcta manipulación del nitrógeno líquido. Con respecto a las ventajas de llevar a cabo el experimento en el aula de clase, es importante considerar que, aunque la explicación de dicho fenómeno se basa en la mecánica cuántica, es posible observar el fenómeno, interactuar con los elementos y describir, a partir del fenómeno macroscópico, lo que ocurre a nivel atómico con el material.

Por otro lado, para poder explicar la levitación magnética, el diamagnetismo perfecto, la resistencia nula y la conductividad infinita de los materiales a bajas temperaturas, también es importante retomar ciertos temas de la física clásica que son subyacentes a las teorías más actuales, particularmente aquellos que pertenecen a la rama del electromagnetismo. Esto hace que la superconductividad cumpla con el segundo criterio expuesto: no estar desvinculado de los estándares propuestos por el Ministerio de Educación Nacional Colombiano, entre los cuales se encuentran:

- Verifico la acción de fuerzas electrostáticas y magnéticas y explico su relación con la carga eléctrica.

- Establezco relaciones entre fuerzas macroscópicas y fuerzas electrostáticas.

- Establezco relaciones entre campo gravitacional y electrostático y entre campo eléctrico y magnético.

- Relaciono diferencia de potencial y corriente con los diferentes elementos de un circuito eléctrico complejo y para todo el sistema.

Como se puede observar, dichos estándares apuntan a la comprensión de temas que pertenecen al electromagnetismo, sin los cuales la explicación que dan los estudiantes alrededor del fenómeno de la superconductividad quedaría incompleta.

Finalmente, el tercer criterio menciona la relevancia del tema para ser enseñado en la escuela media como tópico de física contemporánea, el cual se puede cumplir siempre y cuando se diseñe un método apropiado de enseñanza que incluya un análisis adecuado de los resultados obtenidos por parte de los estudiantes, para 
que se pueda observar la pertinencia de abordar temas de física más avanzada en un curso de secundaria. Razón por la cual, es importante mencionar el por qué se elige un enfoque determinado de enseñanza para abordar una estrategia de aula que dé paso a la enseñanza de la superconductividad.

\section{Aspectos Físicos}

A la luz del conocimiento actual, la superconductividad es una manifestación de un nuevo estado de la materia: el estado superconductor [3], el cual es posible observar en la cotidianidad mediante aplicaciones tecnológicas y en el laboratorio con materiales específicos que muestran fenómenos como la levitación magnética de imanes sobre materiales enfriados a ciertas temperaturas, que se produce por la expulsión de las líneas de campo magnético del interior del material, dando paso a una manifestación física conocida como corrientes persistentes y que a su vez permiten la redistribución de flujo magnético en el superconductor generando que este se comporte como una substancia diamagnética perfecta.

El fenómeno de la superconductividad se descubrió en el experimento que desarrolló Kamerlingh Onnes en el año 1911 luego de haber logrado la licuefacción del helio. Particularmente, su estudio se centró en observar cómo variaba la resistencia eléctrica del mercurio en función de la temperatura, encontrando que a medida que disminuye la temperatura a la que estaba sometido el mercurio, menor era su resistencia eléctrica. Observó además un comportamiento inusual: a la temperatura de $4,2 \mathrm{~K}$ la resistencia de dicho material desciende repentinamente hasta un valor prácticamente nulo, evidenciando una perfecta conducción de la electricidad.

A este descubrimiento se sumaron las investigaciones realizadas por los físicos Walther Meissner y Robert Ochsenfeld quienes, hacia el año 1933, determinaron que si se aplica un campo magnético con un valor específico a dos materiales (un conductor ideal y un superconductor) y se disminuye paulatinamente la temperatura de estos, entonces, mientras ésta se halle por encima de su temperatura crítica, ambos materiales permiten la inclusión del campo magnético en su interior; pero si la temperatura cae por debajo de esa temperatura crítica, se expulsa el campo magnético del interior del material superconductor, mientras que en el material conductor normal se mantiene el campo magnético en su interior. Lo anterior se conoce como el Efecto MeissnerOchsenfeld y es una de las propiedades más importantes que definen el fenómeno de la superconductividad.

A partir del Efecto Meissner [4, es posible observar que cuando un material se encuentra en el estado superconductor en presencia de un campo magnético externo diferente de cero, el superconductor genera su propio campo magnético a través de las corrientes superconductoras, el cual se opone al campo externo, de modo que el valor resultante del campo magnético neto en el interior del material superconductor sea siempre igual a cero. Sin embargo, al aumentar el valor de este campo aplicado, hasta llegar a un valor crítico Bc, los materiales superconductores experimentan dos tipos de transiciones al estado normal. En algunos casos la transición es repentina y abrupta, es decir pasa de no tener campo magnético en su interior a dejar pasar todo el campo aplicado. A los materiales que experimentan este tipo de transición se les conoce como superconductores de tipo I. En otros materiales, la transición para que el material deje penetrar por completo el campo aplicado se da de forma creciente desde un campo magnético Bc1 hasta un campo Bc2, es decir que a partir de cierto valor inicial el campo magnético aplicado, va penetrando el material superconductor y en un valor final deja ingresar por completo el campo aplicado. Los materiales que experimentan esta transición paulatina al estado normal se conocen como superconductores de tipo II.

A la clasificación de los superconductores, teniendo en cuenta el valor de la temperatura crítica y el campo magnético crítico, se sumaron las explicaciones teóricas teniendo en cuenta la mecánica cuántica. Los físicos John Bardeen, Leon Cooper y John Schrieffer (1957) fueron quienes explicaron las interacciones microscópicas de los materiales superconductores a partir de la Teoría BCS [5], motivo por el que recibieron el Premio Nobel de Física en 1972. La teoría BCS expone que dos electrones en el material superconductor son capaces de formar un par ligado llamado par de Cooper. Esos pares de Cooper que se forman por dos electrones, se comportan de forma atractiva entre ellos (a pesar de tener cargas iguales) porque interactúan a través de la red cristalina de los superconductores formada por iones.

Otro de los protagonistas que hizo parte del desarrollo de la superconductividad fue Brian Josephson, al cual se le atribuye el descubrimiento del fenómeno de tunelaje electrónico en estos materiales [6]. Josephson menciona la aparición de un flujo de corriente entre dos materiales superconductores que se encuentran separados por una capa delgada hecha de un material aislante. Por este descubrimiento, Josephson recibe el premio Nobel de Física en el año 1973, y sus investigaciones dan paso al desarrollo de avances científicos relacionados con este fenómeno, por ejemplo, la fabricación de materiales cerámicos (que se caracterizan por no ser materiales conductores ni aislantes de la electricidad) que tuvieran las propiedades de los superconductores, pero que alcanzaran el estado superconductor a temperaturas más elevadas; la teoría de la superconductividad en campos magnéticos intensos y la superfluidez, las cuales fueron presentadas por el físico experimental Alexei Abrikosov y los físicos teóricos Vitali Ginzburg y Anthony Leggett [7], quienes fueron galardonados con el premio Nobel de Física en el año 2003. Las investigaciones sobre superconductores cerámicos con elevada temperatura crítica [8] (originalmente alrededor de los 30K, y superando en la actualidad los $130 \mathrm{~K}$, y $200 \mathrm{~K}$ para ciertos 
materiales a altísimas presiones) estuvieron a cargo del físico Alex Müller y del químico Georg Bednorz, quienes recibieron el premio Nobel de Física en 1987.

Aunque la teoría BCS, explica muy bien el comportamiento de los superconductores convencionales tipo I y tipo II, presenta algunas inconsistencias con respecto a los superconductores de altas temperaturas, dos de los ejemplos de este tipo de superconductores, son aquellos que están formados por óxido de cobre, hierro, entre otros, los cuales hacen parte de las investigaciones actuales sobre este fenómeno.

\section{Aspectos Pedagógicos}

La Enseñanza para la Comprensión (EpC), es un enfoque pedagógico que propone que los estudiantes sean los protagonistas en la educación, de manera que todas las actividades realizadas se centren en lograr que ellos aprendan significativamente los conceptos propuestos [9]. A diferencia de otras perspectivas pedagógicas, el enfoque de la EpC justifica que el objetivo principal de las actividades que se lleven a cabo en el aula es el de proveer a los estudiantes de habilidades que garanticen que realmente alcanzan niveles de comprensión superiores en los temas abordados. La comprensión se define como la flexibilidad del conocimiento que deja en evidencia que los estudiantes entienden, argumentan, discuten y proponen acciones que reflejan que realmente las temáticas son significativas para ellos.

Una ventaja que tiene la EpC, sobre otros modelos pedagógicos es que ofrece una estructura para la organización de las clases que se llevan a cabo con los estudiantes, donde el maestro puede establecer los objetivos de la clase, crear las actividades de la clase y a su vez, de acuerdo con los resultados obtenidos de los estudiantes, hacer la evaluación de la clase a partir de unos criterios de evaluación que trascienden el plano conceptual, dando importancia a las habilidades adquiridas en función del conocimiento recibido.

La enseñanza para la comprensión ofrece cuatro pilares fundamentales, que precisamente dan al maestro una guía para planear, tomando como referencia lo que quiere que sus estudiantes aprendan. El primero de ellos es el tópico generativo, que hace referencia al concepto que se pretende enseñar a los estudiantes. Este tópico debe ser llamativo para ellos, para que se logre captar su atención y realicen las actividades no en función de una calificación sino en función del interés en aprender más acerca del tema. Además, se tienen las metas de comprensión, las cuales se crean para la implementación de la estrategia de forma general y para cada una de las actividades que se realicen en el aula. Estas metas de comprensión de las clases deben responder al logro puntual que se pretende que los estudiantes alcancen, también deben apuntar a cumplir la meta de comprensión abarcadora, la cual pretende que los estudiantes comprendan el fenómeno de la superconductividad.
Otro de los pilares, hace alusión a la evaluación diagnóstica continua, que se encarga de clasificar a los estudiantes desde cuatro dimensiones diferentes: contenidos, métodos, propósitos y formas de comunicación. Finalmente, el pilar que corresponde a cada una de las actividades que se plantean para lograr alcanzar la meta de comprensión, se conoce como desempeños de comprensión, los cuales deben ser claros y explícitos para los estudiantes, generando interés por los temas que se abordan en el aula de clases.

A partir de los desempeños de comprensión, entra a jugar un papel fundamental la realización de prácticas experimentales, ya que se ha demostrado que el trabajo práctico resulta una estrategia educativa útil para conseguir casi cualquier objetivo educativo planteado. Por esta razón, es fundamental realizar experimentos que permitan que los estudiantes hagan una construcción del conocimiento alrededor del tema que se aborda en el aula. Por otro lado, la experimentación permite que los alumnos puedan comprobar las teorías o conceptos que se trabajan en la clase de física, abriendo la posibilidad de mostrar aplicaciones tangibles a los estudiantes cuyo objetivo principal sea despertar su curiosidad e interés.

\section{Diseño y Ejecución}

La estrategia de aula se llevó a cabo con 31 estudiantes del último año del Colegio Bilingüe Clermont, en el año 2020. Estos estudiantes se caracterizaron por ser receptivos, participativos, interesados en su aprendizaje, responsables, seguros de sí mismos, innovadores, reflexivos y respetuosos; cualidades que se hacen evidentes en todas las clases a las cuales asisten. La meta de comprensión abarcadora planteada para cumplir mediante el desarrollo de la implementación fue: Los estudiantes comprenderán el fenómeno de la superconductividad a partir de la puesta en práctica de experimentos que los ayuden a entender conceptos de la física clásica y contemporánea. Además, comprenderán la relación entre el fenómeno de la superconductividad, los avances tecnológicos actuales y las implicaciones sociales que trae el desarrollo de la ciencia en los diferentes contextos mundiales.

Para cumplir esta meta de comprensión, fue necesario crear 3 metas que correspondían a cada una de las unidades que se desarrollaron en el aula, las cuales fueron: 1. ¿Cuáles son los principios físicos clásicos, desde el campo del electromagnetismo, que son necesarios para explicar el fenómeno de la superconductividad? Los alumnos comprenderán conceptos del electromagnetismo a partir de actividades experimentales. 2. ¿Cómo se puede entender microscópicamente el fenómeno de la superconductividad? Los alumnos comprenderán los conceptos de la mecánica cuántica necesarios a partir de actividades experimentales, particularmente con el uso de simulaciones. 3. ¿Cómo se puede explicar el fenómeno de la superconductividad desde los principios físicos 
clásicos y contemporáneos? y ¿qué relación presenta este fenómeno con el mundo actual? Los alumnos comprenderán el fenómeno de la superconductividad a partir de algunos conceptos de la física clásica y contemporánea y logran conectar sus conocimientos con aplicaciones presentes en la actualidad.

A partir de estas metas de comprensión, es posible notar la relación de cada una con tres etapas específicas planteadas para llevar a cabo la implementación en el aula: la primera corresponde a los fundamentos electromagnéticos del fenómeno de la superconductividad, la segunda corresponde a los fundamentos cuánticos del fenómeno y la tercera, establece la relación de los dos primeros para poder dar cuenta del fenómeno de la superconductividad y su relación con el mundo contemporáneo. En la Tabla 1 se presenta un esquema general de las clases, evidenciando los conocimientos previos de los estudiantes antes de realizar las actividades propuestas y la puesta en práctica central que se llevó a cabo durante la implementación.

Como se puede observar en la Tabla 1 en las primeras 4 clases la intención era que los estudiantes tuvieran claros los conceptos del electromagnetismo. Por tal razón se estudiaron los siguientes tópicos generativos, que hacen parte de la etapa de desempeños preliminares: carga eléctrica y sus manifestaciones; superficies equipotenciales y campo eléctrico; resistencia, resistividad y variación de la resistencia con la temperatura; introducción al

Tabla 1: Esquema general de la secuencia didáctica que se llevó a cabo en la implementación en el aula.

\begin{tabular}{|c|c|c|}
\hline Clase & Conocimientos previos & Actividad central \\
\hline $\begin{array}{l}\text { 1. Carga eléctrica y } \\
\text { sus manifestaciones. }\end{array}$ & $\begin{array}{l}\text { Los estudiantes tienen conocimientos de } \\
\text { cinemática, dinámica, energía. Cuentan con } \\
\text { algunas nociones de electrostática. }\end{array}$ & $\begin{array}{l}\text { Los estudiantes desarrollaron un laboratorio exploratorio, } \\
\text { en el cual observaron algunas manifestaciones de las } \\
\text { cargas eléctricas. }\end{array}$ \\
\hline $\begin{array}{l}\text { 2. Superficies } \\
\text { equipotenciales y } \\
\text { campo eléctrico. }\end{array}$ & $\begin{array}{l}\text { Los estudiantes tienen claridad en los } \\
\text { conceptos relacionados con la carga eléctrica } \\
\text { y la forma como ésta se transfiere. }\end{array}$ & $\begin{array}{l}\text { La clase inició con una simulación, en la cual se } \\
\text { evidenciaba la diferencia entre campo y potencial } \\
\text { eléctricos de forma visual. Luego llevaron a cabo una } \\
\text { práctica experimental donde realizaron mediciones de } \\
\text { diferencia de potencial para establecer su relación con el } \\
\text { campo eléctrico. }\end{array}$ \\
\hline $\begin{array}{l}\text { 3. Resistencia, } \\
\text { resistividad y } \\
\text { variación de la } \\
\text { resistencia con la } \\
\text { temperatura. }\end{array}$ & $\begin{array}{l}\text { Los estudiantes reconocen la existencia del } \\
\text { campo eléctrico como una perturbación en el } \\
\text { espacio debida a las cargas eléctricas. }\end{array}$ & $\begin{array}{l}\text { En primer lugar, los estudiantes realizaron mediciones de } \\
\text { diferencia de potencial y corriente sobre resistencias, para } \\
\text { determinar expermentalmente la ley de Ohm. Y en } \\
\text { segundo lugar observaron una simulación relacionada con } \\
\text { los temas propuestos. }\end{array}$ \\
\hline $\begin{array}{l}\text { 4. Introducción al } \\
\text { magnetismo. }\end{array}$ & $\begin{array}{l}\text { Los estudiantes tienen conocimiento de las } \\
\text { cargas eléctricas, los campos que se generan } \\
\text { a partir de éstas, los circuitos eléctricos, la } \\
\text { relación entre corriente, voltaje y resistencia } \\
\text { y cómo la resistencia y la resistividad varían } \\
\text { con la temperatura. }\end{array}$ & $\begin{array}{l}\text { Los estudiantes llevaron a cabo las actividades de } \\
\text { magnetismo donde observaron con limadura de hierro el } \\
\text { campo magnético alrededor de los imanes. Además } \\
\text { complementaron dicha práctica con algunas preguntas } \\
\text { propuestas en un simulador. }\end{array}$ \\
\hline $\begin{array}{l}\text { 5. Explicación de la } \\
\text { superconductividad }\end{array}$ & $\begin{array}{l}\text { Los estudiantes identifican las propiedades } \\
\text { de los imanes, incluyendo la manifestación } \\
\text { del campo magnético. }\end{array}$ & $\begin{array}{l}\text { Los estudiantes llevaron a cabo las actividades } \\
\text { introductorias, en las cuales podían observar el efecto } \\
\text { Meissner a partir de simuladores. }\end{array}$ \\
\hline $\begin{array}{l}\text { 6. Tipos de } \\
\text { superconductores y } \\
\text { vórtices }\end{array}$ & $\begin{array}{l}\text { Los estudiantes tienen conocimientos } \\
\text { relacionados con el efecto Meissner. Tienen } \\
\text { nociones sobre diamagnetismo perfecto, } \\
\text { Estado Meissner mixto y diferencia entre el } \\
\text { superconductor y el diamagnético perfecto. }\end{array}$ & $\begin{array}{l}\text { Se mostró a los estudiantes una presentación en power } \\
\text { point en la cual se apreciaban las características físicas y } \\
\text { las diferencias entre cada tipo de superconductor. Además } \\
\text { se presentó información acerca de la definición de los } \\
\text { vórtices en los materiales en estado superconductor. }\end{array}$ \\
\hline 7. Pares de Cooper. & $\begin{array}{l}\text { Los estudiantes además del efecto Meissner, } \\
\text { reconocen los tipos de superconductores y } \\
\text { sus características principales. }\end{array}$ & $\begin{array}{l}\text { En grupos de trabajo los estudiantes realizaron una } \\
\text { lectura, en la cual se hace una presentación de los pares } \\
\text { de Cooper a partir de gráficos y explicaciones sencillas. }\end{array}$ \\
\hline 8. Experimento & $\begin{array}{l}\text { Los estudiantes pueden explicar el } \\
\text { comportamiento de los superconductores a } \\
\text { partir de los pares de Cooper. }\end{array}$ & $\begin{array}{l}\text { Los estudiantes observan el fenómeno de } \\
\text { superconductividad y generan algunas conclusiones sobre } \\
\text { el tipo de superconductor con el cual se está realizando el } \\
\text { experimento, los campos magnéticos, la relación de la } \\
\text { resistividad con la temperatura y el efecto } \\
\text { Meissner-Ochsenfeld. }\end{array}$ \\
\hline 9. Actividad final & $\begin{array}{l}\text { Los estudiantes son capaces de explicar el } \\
\text { fenómeno de la superconductividad a partir } \\
\text { de la atracción y repulsión de cargas } \\
\text { eléctricas, cambio de la resistencia en función } \\
\text { de la temperatura, efecto } \\
\text { Meissner-Ochsenfeld, campos magnéticos, } \\
\text { tipos de superconductores y pares de Cooper. }\end{array}$ & $\begin{array}{l}\text { Los estudiantes presentan los trabajos realizados a sus } \\
\text { compañeros, teniendo en cuenta la relación de uno de los } \\
\text { temas vistos durante la estrategia didáctica con la } \\
\text { superconductividad. }\end{array}$ \\
\hline 10. Aplicaciones & & $\begin{array}{l}\text { Los estudiantes presentan a sus compañeros la } \\
\text { información consultada acerca de las aplicaciones de la } \\
\text { superconductividad en contextos actuales. }\end{array}$ \\
\hline
\end{tabular}


magnetismo. Además, para cumplir con las metas de comprensión de esta unidad, los desempeños de comprensión que se diseñaron estaban explícitos en 4 guías tomadas y adaptadas de Reyes, Salcedo \& Carrillo [10], desde las cuales los estudiantes debían llevar a cabo una serie de actividades experimentales que desarrollaron grupalmente.

Por otro lado, en las siguientes tres clases, se buscó que los estudiantes cumplieran la segunda meta de comprensión, de manera que se establecieron otros tres tópicos generativos que hicieron parte de la etapa de investigación guiada: explicación de la superconductividad; tipos de superconductores y vórtices; pares de Cooper. Para el primer tópico generativo, los estudiantes observaron una serie de simulaciones desde las cuales contrastan sus ideas intuitivas con el nuevo conocimiento que se presentaba a partir de actividades prácticas. Además, para el desarrollo del segundo tópico, se realizó una explicación magistral en donde se presenta a los estudiantes las diferencias fundamentales entre los superconductores tipo I y II, dando paso así a la comprensión del Efecto Meissner - Ochsenfeld y del estado vórtice. Finalmente, para el último tópico generativo, se propuso como desempeño de comprensión el análisis de la lectura de Pares de Cooper disponible en la página de cuentos cuánticos 11] desde la cual los estudiantes pudieron analizar las particularidades que se presentan en los materiales cuando se encuentran en el estado superconductor.

En la última etapa, que corresponde al proyecto final de síntesis, se desarrollaron tres tópicos generativos: el experimento de superconductividad; explicación de la superconductividad desde la física clásica y contemporánea; y aplicaciones. En la clase número 8, en la cual se realizó el experimento los estudiantes pudieron observar el fenómeno de levitación magnética desde el cual inician con la descripción del fenómeno desde una mirada electromagnética y cuántica. En la penúltima clase, realizan una presentación creativa evidenciando su comprensión alrededor del fenómeno de la superconductividad. Y en la última clase, los estudiantes hacen una discusión alrededor de las aplicaciones de la superconductividad en el mundo contemporáneo y cómo estas se han empleado para mejorar la vida de las personas en diferentes aspectos de la vida cotidiana.

\section{Resultados}

Teniendo en cuenta que la intención de la estrategia de aula era lograr que los estudiantes comprendieran el fenómeno de la superconductividad, que corresponde a un tópico de la física contemporánea, se presentan los resultados de la evaluación diagnóstica continua que se ejecutó en el aula desde la cual es posible observar los niveles de comprensión alcanzados por los estudiantes en cada una de las clases, que se evalúan de acuerdo a las cuatro dimensiones de comprensión que se presentan desde la Enseñanza para la Comprensión.

Para las clases que corresponden a los desempeños preliminares, se evalúan los criterios que se observan en la Tabla 2, donde se presenta el porcentaje de estudiantes que alcanzan cada uno de los niveles de comprensión siendo 0 el nivel que corresponde a la no realización de la actividad, 1 el nivel que muestra comprensión más elemental y 4 el nivel de comprensión superior.

En la Tabla 2 se puede observar que para cada una de las clases, se evaluaron diferentes dimensiones y a su vez diferentes criterios que están estrechamente relacionados con los desempeños de comprensión, es decir con las actividades que se llevaron a cabo con los estudiantes. Como se había mencionado anteriormente, las actividades experimentales fueron fundamentales en esta etapa de implementación, particularmente se hizo uso del generador de Van der Graff para dar cuenta de la carga eléctrica; limadura de hierro e imanes, para entender el campo magnético; multímetros, resistencias y electrodos, para medir la diferencia de potencial y determinar las características de la variación de la resistencia con la temperatura. Además de las prácticas de laboratorio que realizaron los estudiantes, las sesiones estuvieron acompañadas de discusiones previas a las actividades, en las cuales los estudiantes hablaban acerca de lo que sabían de cada uno de los temas, el uso de analogías y la implementación de algunas simulaciones, específicamente de Phet [12] y Supercomet [13].

Tabla 2: Evaluación diagnóstica continua de la etapa preliminar, donde se abordan los temas del electromagnetismo.

\begin{tabular}{|c|c|c|c|c|c|c|c|c|c|c|c|c|c|c|c|c|c|c|c|c|c|}
\hline \multirow[t]{2}{*}{ Dimensión } & \multirow{2}{*}{$\begin{array}{c}\text { Criterio/clase } \\
\text { Niveles de comprensión (\%) }\end{array}$} & \multicolumn{5}{|c|}{ Carga eléctrica } & \multicolumn{3}{|c|}{$\begin{array}{l}\text { Campo } \\
\text { eléctrico }\end{array}$} & \multicolumn{3}{|c|}{ Resistencia } & \multicolumn{4}{|c|}{ Magnetismo } & \multicolumn{5}{|c|}{$\begin{array}{l}\text { Resistencia vs } \\
\text { Temperatura }\end{array}$} \\
\hline & & 0 & 1 & 2 & 3 & 4 & 2 & 3 & 4 & 2 & 3 & 4 & 1 & 2 & 3 & 4 & 0 & 1 & 2 & 3 & 4 \\
\hline Contenidos & Ideas intuitivas transformadas & 6 & 13 & 32 & 26 & 23 & 13 & 52 & 35 & 13 & 13 & 74 & 3 & 16 & 49 & 32 & 3 & 3 & 33 & 32 & 29 \\
\hline \multirow{3}{*}{ Propósitos } & Multiples usos del conocimiento & 7 & 0 & 26 & 48 & 19 & 13 & 58 & 29 & & & & & & & & & & & & \\
\hline & Conciencia de los propósitos del conocimiento & & & & & & & & & 13 & 0 & 87 & & & & & & & & & \\
\hline & Buen Manejo y autonomía & & & & & & & & & & & & 0 & 16 & 55 & 29 & & & & & \\
\hline \multirow{2}{*}{ Métodos } & Construir conocimiento dentro del dominio & 7 & 6 & 29 & 29 & 29 & & & & 13 & 26 & 61 & & & & & & & & & \\
\hline & Sano escepticismo & & & & & & 39 & 22 & 39 & & & & 3 & 13 & 39 & 45 & & & & & \\
\hline \multirow{2}{*}{$\begin{array}{l}\text { Formas de } \\
\text { comunicación }\end{array}$} & Consideración de la audiencia y el contexto & & & & & & 0 & 48 & 52 & & & & & & & & & & & & \\
\hline & Efectivo uso de sistemas de símbolos & & & & & & & & & 0 & 26 & 74 & & & & & & & & & \\
\hline
\end{tabular}


A partir de los resultados que se presentan de forma sintetizada en la Tabla 2 se puede concluir que a los estudiantes se les facilita expresar sus ideas, razón por la cual los niveles más altos de comprensión se encuentran en la dimensión de métodos de comunicación. Además, se puede observar que en estas clases, prevalecen las ideas intuitivas de los estudiantes, ya que no en todas las clases la mayoría de los estudiantes alcanza niveles de comprensión 3 y 4 . Finalmente, se hace evidente que los estudiantes, hacen uso de las prácticas experimentales para la construcción del conocimiento, lo cual se puede evidenciar en las conclusiones que daban en cada una de las hojas de actividad de cada clase.

Por otro lado, se tienen las clases que hacen parte de la etapa de investigación guiada, las cuales corresponden específicamente al tópico de la superconductividad. La primera de ellas se ejecutó alrededor de la comprensión del efecto Meissner, para esta clase se utilizó la simulación de Supercomet [13] acompañada de una hoja de actividad en la cual el estudiante consignaba su proceso, a través del cual fue posible observar el contraste entre sus ideas intuitivas y lo aprendido a partir de la simulación. Uno de los grupos de trabajo concluyó "El efecto Meissner-Ochsenfeld es una propiedad de los superconductores. Este efecto consiste en la expulsión completa del campo magnético cuando los superconductores se enfrían por debajo de su temperatura crítica. Por otro lado, si está por encima de su temperatura crítica, el campo magnético atraviesa los superconductores".

En la segunda clase, que hizo parte de esta etapa se abordó el tópico generativo de tipos de superconductores y estado vórtice. A diferencia de las clases anteriores, en esta ocasión se recurre a realizar una clase tradicional en la cual la docente explicó a sus estudiantes las características de los tipos de superconductores y las diferencias que se presentan entre los dos. Y después por grupos de trabajo los estudiantes presentan sus conclusiones alrededor del tema, por ejemplo, a continuación se menciona la conclusión que escribe uno de estos grupos: "Para comenzar, todos los superconductores tipo 1 son elementos puros. Además, en este tipo de superconductor, los campos magnéticos se repelen totalmente. En consecuencia, el imán puede levitar. Además, una característica importante de los superconductores tipo 1 es que estos suelen pasar del estado superconductor al estado normal de manera brusca. Adicionalmente, en los superconductores tipo uno nunca se generan vórtices"

Por otro lado, el tópico de Pares de Cooper se trabajó en el aula a partir de una lectura llamada Cooper, con un par 11, la cual describe el comportamiento de los electrones e iones de los materiales cuando estos se encuentran en el estado superconductor. En la lectura, aparecían algunos conceptos que hacen parte de la física moderna y contemporánea, por ejemplo fermiones, principio de exclusión de Pauli, bosones, etc., los cuales generaron que los estudiantes investigaran alrededor de estos temas, para poder entender y dar cuenta de su comprensión sobre el fenómeno de la superconductividad. A continuación, se muestra una de las conclusiones escritas por un grupo de trabajo "En la red metálica están los iones y los electrones, cuando hay una baja temperatura hay menor energía cinética y por lo tanto los iones se quedan quietos donde están y los electrones...", "... pueden pasar libremente porque los iones están quietos entonces no los afectan". "Si, en cambio, hay más temperatura entonces se empiezan a mover y los pares de Cooper no se van a poder formar. Otra cosa es que los pares de Cooper son muy débiles, entonces si cambia la temperatura se afectan."

En la Tabla 3, se muestran los resultados de los niveles de comprensión que alcanzaron los estudiantes en cada una de las clases, donde se puede apreciar que ningún estudiante obtuvo el nivel más bajo de comprensión, lo cual indica que los temas relacionados en la explicación del fenómeno desde la física moderna y contemporánea quedó claro para ellos. Además, es posible observar que la mayoría de los estudiantes ocupan el máximo nivel de comprensión en los tópicos de Efecto Meissner y Pares de Cooper, y todos los estudiantes evidenciaron comprensión al momento de dar a conocer sus ideas, lo que los posicionó en el nivel máximo de comprensión en la dimensión de formas de comunicación.

Con el tópico de Pares de Cooper, se dio por finalizada la construcción teórica desde el electromagnetismo clásico y la física contemporánea para que los estudiantes den cuenta del fenómeno propuesto. De tal manera que, en la siguiente clase, se realiza con los estudiantes el experimento de la superconductividad, en el cual se hace evidente la levitación magnética del imán sobre el material cerámico BSCCO que, al ser enfriado con

Tabla 3: Evaluación diagnóstica continua de la etapa de investigación guiada, donde se abordan los temas de superconductividad.

\begin{tabular}{|c|c|c|c|c|c|c|c|c|c|}
\hline \multirow[t]{2}{*}{ Dimensión } & \multirow{2}{*}{$\begin{array}{c}\text { Criterio/clase } \\
\text { Niveles de comprensión (\%) }\end{array}$} & \multicolumn{3}{|c|}{ Efecto Meissner } & \multicolumn{2}{|c|}{$\begin{array}{l}\text { Tipos } \\
\text { de S.C }\end{array}$} & \multicolumn{3}{|c|}{$\begin{array}{c}\text { Pares de } \\
\text { Cooper }\end{array}$} \\
\hline & & 2 & 3 & 4 & 3 & 4 & 2 & 3 & 4 \\
\hline \multirow{2}{*}{ Contenidos } & Ideas intuitivas transformadas & 13 & 0 & 87 & & & 13 & 48 & 39 \\
\hline & Redes conceptuales coherentes y ricas & & & & 10 & 90 & & & \\
\hline Propósitos & Buen Manejo y autonomía & 13 & 0 & 87 & 13 & 87 & & & \\
\hline \multirow{2}{*}{ Métodos } & Construir conocimiento dentro del dominio & 29 & 29 & 29 & & & 0 & 0 & 100 \\
\hline & Sano escepticismo & 12 & 11 & 77 & & & & & \\
\hline Formas de comunicación & Dominio de los géneros de realización & 0 & 0 & 100 & 0 & 100 & 0 & 0 & 100 \\
\hline
\end{tabular}


nitrógeno líquido, cambia sus características físicas, lo que genera que se encuentre en la fase superconductora. Aunque en esta clase, no se realizó evaluación, surgieron discusiones mientras se realizaba el experimento que permitieron concluir que los estudiantes eran capaces de explicar desde el electromagnetismo y desde la física contemporánea lo que estaban observando.

En la tercera y última etapa de la implementación, se realizaron dos clases fundamentales como cierre. La primera estaba relacionada con las aplicaciones de la superconductividad en el mundo contemporáneo, que al igual que la clase del experimento, no tuvo una evaluación diagnóstica continua, teniendo en cuenta que el objetivo era que los estudiantes tuvieran cierta libertad en cuanto a la temática que quisieran abordar. Para esta discusión, se realizó un conversatorio en el cual los estudiantes presentaban a sus compañeros cómo el fenómeno de la superconductividad se ha empleado en el campo de la electrónica, la física de partículas, la medicina y el transporte, para mejorar ciertos aspectos fundamentales de la vida de las personas en el mundo actual.

Finalmente, se concluye la implementación con una actividad final, en la cual los estudiantes debían explicar el fenómeno de la superconductividad teniendo en cuenta todo lo que se estudió en las diferentes clases. Cada grupo de trabajo debía encontrar una forma creativa de presentar a sus compañeros aspectos fundamentales de la superconductividad que demuestren comprensión alrededor del fenómeno. Los estudiantes escribieron cuentos, crearon videos, inventaron juegos, presentaron obras de teatro, maquetas y canciones, para dar cuenta de su conocimiento alrededor del fenómeno de la superconductividad, detallando cada tópico trabajado a lo largo de la implementación. En la Tabla 4 se puede apreciar la evaluación de esta actividad de cierre. Como se puede observar, en las dimensiones de propósitos, métodos y formas de comunicación los estudiantes alcanzan el nivel superior, lo cual refleja que lograron cumplir la meta de comprensión abarcadora durante la implementación. En la dimensión de contenidos el $74 \%$ obtiene nivel superior mientras que el $26 \%$ de los estudiantes obtienen niveles 2 y 3 , particularmente por falta de profundización en los contenidos.
Al finalizar la implementación, los estudiantes realizan una encuesta en la cual evalúan diferentes aspectos de la estrategia de aula que se llevó a cabo para que ellos comprendieran un tópico de la física contemporánea, ellos debían medir su nivel de satisfacción en 10 aspectos diferentes, siendo 1 poco satisfecho y 5 muy satisfecho. Los aspectos evaluados fueron los siguientes: Estructura de la clase: parte teórica y experimental; claridad en las instrucciones; evaluación de las actividades desde las 4 dimensiones de EpC; trabajo en grupo; simulaciones empleadas; actividades experimentales realizadas; comprensión de los conceptos de física clásica: electricidad y magnetismo; comprensión de conceptos avanzados de física: Efecto Meissner, vórtices, tipos de superconductores y pares de Cooper; comprensión del fenómeno superconductividad a partir de conceptos de física clásica y contemporánea; guía de la profesora para la comprensión del fenómeno de la superconductividad.

Desde la perspectiva de los estudiantes, la implementación fue relativamente exitosa en todos aspectos, ya que más del $50 \%$ de los estudiantes eligió la opción de mayor nivel en los 10 aspectos. Sin embargo, obtener niveles de satisfacción 2, 3 y 4 en algunos de los puntos para los estudiantes, por ejemplo en los aspectos de comprensión de los conceptos de física clásica: electricidad y magnetismo y comprensión de conceptos avanzados de física: Efecto Meissner, vórtices, tipos de superconductores y pares de Cooper, abre la posibilidad de evaluar las acciones que se llevaron a cabo durante la implementación, de manera que en futuras estrategias de enseñanza de la física contemporánea en el nivel medio, en las cuales se consideren estos mismos aspectos, se pueda obtener nivel máximo de satisfacción de todos los estudiantes.

Es interesante señalar que, aunque la sistematización de la información muestre resultados positivos alrededor de unos criterios de evaluación, siempre es importante generar auto-evaluaciones, desde las cuales se pueda apreciar cómo se percibe así mismo el estudiante en cuanto a la comprensión de las temáticas estudiadas. Esto se menciona porque los dos aspectos que presentan menor cantidad de estudiantes con un nivel superior de satisfacción son los relacionados con la comprensión de los conceptos físicos trabajados en la experiencia lo cual

Tabla 4: Evaluación diagnóstica continua de la etapa de proyecto final de síntesis, donde se aborda la comprensión del fenómeno de la superconductividad desde la mirada clásica y contemporánea.

\begin{tabular}{|c|c|c|c|c|}
\hline Dimensión & Criterio/clase & \multicolumn{3}{|c|}{ Actividad final } \\
\hline & Niveles de comprensión (\%) & 2 & 3 & 4 \\
\hline \multirow[t]{2}{*}{ Contenidos } & Ideas intuitivas transformadas & \multirow{2}{*}{13} & \multirow{2}{*}{13} & \multirow{2}{*}{74} \\
\hline & Redes conceptuales coherentes y ricas & & & \\
\hline \multirow{2}{*}{ Propósitos } & Múltiples usos del conocimiento & \multirow{2}{*}{0} & \multirow{2}{*}{0} & \multirow{2}{*}{100} \\
\hline & Conciencia de los propósitos del conocimiento & & & \\
\hline \multirow{2}{*}{ Métodos } & Construir conocimiento dentro del dominio & \multirow{2}{*}{0} & \multirow{2}{*}{0} & \multirow{2}{*}{100} \\
\hline & Validar el conocimiento en el dominio & & & \\
\hline \multirow{2}{*}{ Formas de comunicación } & Consideración de la audiencia y el contexto & \multirow{2}{*}{0} & \multirow{2}{*}{0} & \multirow{2}{*}{100} \\
\hline & Dominio de los géneros de realización & & & \\
\hline
\end{tabular}


no está en concordancia con las respuestas dadas por los estudiantes en las actividades planteadas durante las 10 clases de implementación, ya que a partir de los criterios de evaluación planteados, se evidenció que todos lograron alcanzar niveles 3 y 4 de comprensión en cuanto a la dimensión de contenidos.

Un aspecto positivo de los resultados obtenidos a partir de la encuesta, es que ningún estudiante presenta un nivel de satisfacción bajo en ninguno de los aspectos evaluados y que solo 1 de los 31 estudiantes, considera que no comprendió a la perfección los temas avanzados vistos alrededor del fenómeno de la superconductividad. Por otro lado, los estudiantes consideran que las instrucciones dadas en la clase, el uso de simulaciones y el trabajo en grupo, cumplió con sus expectativas, de modo que se continuará en próximas prácticas pedagógicas con el manejo adecuado de estos aspectos, de manera que posibilite la construcción del conocimiento trabajado en las clases.

\section{Conclusiones}

En este trabajo se presentaron los resultados de una investigación sobre la ejecución de una experiencia de enseñanza de un tópico de física contemporánea, la superconductividad, realizada ante un alumnado de nivel medio. En los distintos capítulos que componen el cuerpo de esta investigación, se desarrollaron en detalle todos los aspectos que han sido necesarios considerar para llevar satisfactoriamente a término esta experiencia educativa experimental, a saber:

1. Selección de un tópico de física contemporánea que se vincula con los Estándares Educativos propuestos por el Ministerio de Educación Nacional Colombiano.

2. Estudio del fenómeno de la superconductividad considerando aspectos teóricos y experimentales, e incluyendo su descripción teórica tanto clásica como cuántica. Por las características del tema seleccionado, esto ha demandado la realización de una extensa búsqueda bibliográfica que incluye una laboriosa tarea de condensación y adaptación de contenidos de textos y artículos de nivel muy avanzado tanto en Física como Matemáticas.

3. Estudio del marco pedagógico sobre el que se basa esta investigación sobre la enseñanza de un tópico de física contemporánea: el enfoque de Enseñanza para la Comprensión.

4. Diseño y desarrollo del plan de acción necesario para llevar a cabo esta experiencia educativa, considerando todos los aspectos prácticos involucrados, y debiendo tomar en cuenta que el estudiantado asiste simultáneamente a otras actividades que también les demandan tiempo y esfuerzo.
5. Reporte sobre los resultados obtenidos, incluyendo la evaluación sobre el impacto que tuvo la experiencia para los estudiantes.

La investigación que se presenta en este trabajo ha mostrado que uno de los aspectos más importantes como docente, es la satisfacción de ver que los estudiantes gozan del trabajo experimental en la clase, se interesan por ampliar sus conocimientos y se sienten motivados por aprender temas nuevos, con la gran expectativa de dejarse sorprender con las maravillas que la física puede ofrecer. Del mismo modo, es fundamental replicar en el futuro esta experiencia pedagógica con otros estudiantes de último año, ya que se abre la posibilidad de enseñar conceptos de electromagnetismo y cuántica, a partir del fenómeno de la superconductividad, acercando a los estudiantes a este tópico de la física contemporánea. También, se abre la posibilidad de explorar otros tópicos de la física actual, que se vinculen con los lineamientos educativos para la enseñanza de la física en la institución y que logren generar motivación en los estudiantes para que comprendan diferentes tópicos de la física contemporánea.

\section{Agradecimientos}

Agradecemos profundamente al Doctor Carlos García Canal (Universidad Nacional de la Plata, Argentina) por sus comentarios y sugerencias durante la realización de este trabajo y a los estudiantes del Colegio Bilingüe Clermont por su responsabilidad, creatividad y constancia.

\section{Referências}

[1] MINISTERIO DE EDUCACIÓN NACIONAL, Estándares de Educación en Ciencias Naturales y Ciencias Sociales. Bogotá, 2004. Disponible en: https://www.mineducacion.gov.co/1759/articles-8103 3_archivo_pdf.pdf.

[2] Superconductividad (ICMM-CSIC), https://wp.icmm. csic.es/superconductividad/.

[3] H. Vucetich, Superconductividad (UNLP, La Plata, 1983).

[4] P.A. Tipler y R.A. Llewellyn, Modern Physics (W.H. Freeman, New York, 2008).

[5] J. Bardeen, L.N. Cooper y J.R. Schrieffer, Physical Review 108, 1175 (1957).

[6] B.D. Josephson, The Discovery of tunnelling supercurrents. 1973. Disponible en: https://www.nobelprize.org /prizes/physics/1973/josephson/lecture/.

[7] V.L. Ginzburg, On superconductivity and Superfluidity. 2003. Disponible en: https://www.nobelprize.org/prize s/physics/2003/ginzburg/lecture/

[8] L.F. Magaña Solís, Los superconductores (Fondo de Cultura Económica, Ciudad de México, 2012).

[9] M. Stone Wiske, La Enseñanza para la Comprensión, vinculación entre la investigación y la práctica (Paidós, Buenos Aires, 1999). 
[10] C.C. Reyes Aldasoro, A. Salcedo González y F. Carrillo Valderrábano, Manual de Experimentos de Electromagnetismo (ITAM, Ciudad de México, 2000).

[11] Cuentos cuánticos, https://cuentos-cuanticos.com/2013 /04/26/par-de-cooper/, accedido en 09/04/2019.

[12] PhET Interactive Simulations, https://phet.colorado.ed $\mathrm{u} /$ sims/html/resistance-in-a-wire/latest/resistance-ina-wire_es.html, accedido en 02/05/2019.

[13] Supercomet, http://online.supercomet.no, accedido en $12 / 02 / 2019$. 